\title{
Investigation of cleaning effect for airport runway lamps by using baking soda powder
}

\author{
Yang Zhao ${ }^{1,2}$, Xiaohong $\mathrm{Ge}^{1,2,{ }^{*}}$, Xing Wang ${ }^{1,2}$, Ke 'an Yue ${ }^{1,2}$, Yi Chen ${ }^{1,2}$, and Jinhuo Wang ${ }^{1,2}$ \\ ${ }^{1}$ Key Laboratory of Functional Materials and Application of Fujian Province, Xiamen University of \\ Technology, Xiamen 361024, China; \\ ${ }^{2}$ Xiamen Engineering and Technology Research Center of High Efficiency and Precision Intelligent \\ Manufacturing, School of Materials Science and Engineering, Xiamen University of Technology, \\ Xiamen 361024, China;
}

\begin{abstract}
In order to ensure the bright effect of airport navigation lights, dry powder cleaning technology was adopted in this paper to improve the efficiency of removing sticky dirt on the lamp surface. The atomization scheme by mixing dry powder with water was designed through deliberately selecting the appropriate injection nozzle structure to reduce impact of powder pollution on the environment. Both simulation analysis and experimental testing showed that the overall cleaning effect and the atomization scheme were feasible, which reduce the dust pollution by more than $90 \%$.
\end{abstract}

\section{Introduction}

In recent years, with rapid development of the aviation industry, the time for cleaning and maintenance of navigation lights at the airport (as shown in Fig.1) has become shorter and shorter. In this paper, the dry powder cleaning method is adopted for research and application.

Dry powder abrasive consists of sodium bicarbonate powder, commonly known as baking soda, which is an alkaline abrasive with water solubility of $7.8 \mathrm{~g}\left(18^{\circ} \mathrm{C}\right)$ in water.

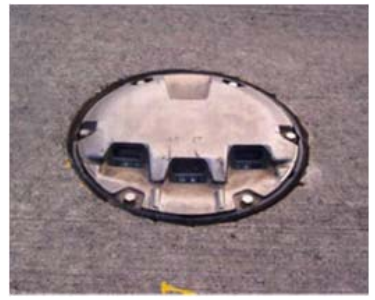

Fig.1. An embedded airport navigation lamp.

Hu Weiwei ${ }^{[1]}$ from Xiamen University of Technology used solid carbonate powder as the cleaning medium, commonly known as a dry powder, and optimized the cleaning parameters. Wang et al. ${ }^{[2]}$ studied the cleaning effect of dry powder cleaning technology on airport

${ }^{*}$ Corresponding author: xhge@xmut.edu.cn 
navigation lights. Gao et al. ${ }^{[3]}$, Cheng ${ }^{[4]}$ also used dry powder cleaning technology successively to study the effect of dry powder cleaning navigation lights.

However, dust and other issues also exist in the process of cleaning, this paper is thus to investigate the cleaning equipment by optimizing water atomized parameters, aiming at improving the airport navigation lights with high cleaning efficiency. At the same time, it is to reduce the harm of dust to the human body in dry powder cleaning.

\section{Mechanism of atomization dust suppression}

The theory of liquid jet atomization in transverse airflow was first proposed by Chen et $\mathrm{al}^{[5]}$. The atomization process mainly includes two aspects: primary crushing process and secondary crushing process. By fluid instability analysis, it can be seen that the liquid jet leaves the nozzle as a complete liquid column. Because of the axial instability, the developed liquid column eventually decomposes into droplets and liquid blocks, which is called the column splitting process. (Fig.2)

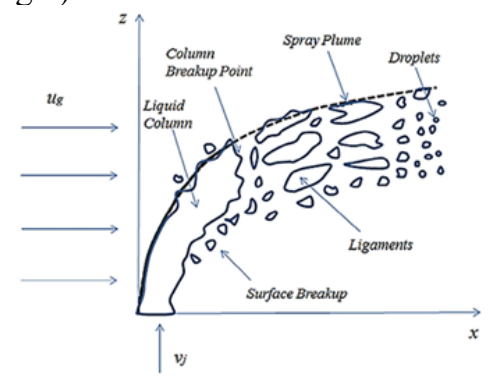

Fig.2. Atomization process of a liquid jet in transverse flow.

The most commonly used liquid jet-breaking standard for transverse airflow is proposed by Ohnesorge ${ }^{[6]}$.

Based on previous studies, Reitz ${ }^{[7]}$ attempted to resolve some of the uncertainties on the Ohnesorge chart. His analysis is based on an interpretation of data from diesel spray. According to the Reitz mechanism, as shown in Fig.3, as the liquid injection rate gradually increases, the following four decomposition modes are obtained: Rayleigh and broken, the first disruption, the second wind induced, and the atomization.

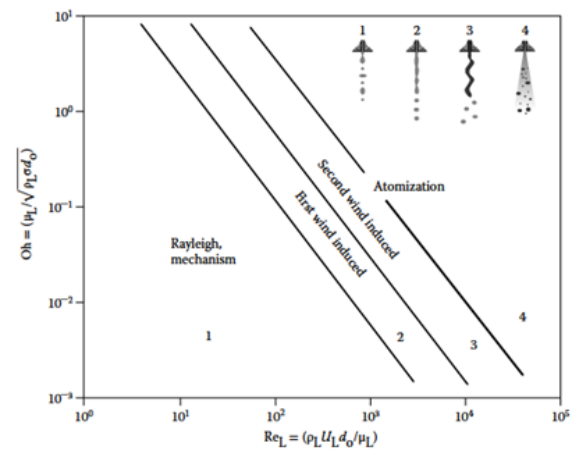

Fig. 3. Reitz mechanism broken. 


\section{Simulation analysis of atomization}

\section{1 atomization modeling and parameter setting}

To simulate the atomization and breakage process and jet characteristics of a liquid jet in transverse airflow, CLSVOF method has been used to study the breakage process and breakage position characteristics of a liquid jet in the transverse airflow. CLSVOF method is combined with Level Set and VOF method. The analysis software Fluent was used to determine the calculation domain and boundary settings inside the nozzle, as shown in Fig.4. The wall surface of the injection flow was set as the non-slip boundary, and the other boundaries were set as the slip boundary. The computational domain grid was divided into unstructured grids with a uniform density distribution. To prevent pseudo-breakage of the liquid phase caused by the inhomogeneity of the computational domain grid, domain size values were calculated as shown in Table 1.

Table 1. Calculate the dimensions of the domains.

Area
Length of the calculation domain

Fig.4. Crushing process simulation study calculation area

Because of the high velocity of transverse airflow, it can be regarded as compressible fluid, so it was calculated based on the pressure solver. The turbulence model adopted the LES model, which can accurately capture the air-liquid interface and check the crushing process. The boundary conditions are shown in Table 2, and the physical parameters remain unchanged in the calculation process.

Table 2. Boundary condition setting of simulation.

\begin{tabular}{cc}
\hline Initial condition & Parameter \\
\hline Air inlet & Pressure inlet: $0.2 \mathrm{MPa}-0.5 \mathrm{MPa}$ \\
Irrigation-water inlet & Pressure inlet: $0.5 \mathrm{MPa}$ \\
Outlet & Pressure outlet: $1.01325 \mathrm{MPa}$ \\
\hline
\end{tabular}

\subsection{Jetting characteristics of a liquid jet in the nozzle}

Injecting depth is an important parameter to evaluate the mixing characteristics of the liquid jet and free air, which can affect the atomization efficiency. The injection depth has a great influence on the liquid jet atomization and the pressure change in the pipe. 
It can be seen from Fig. 5 that the crushing position is affected by the air inlet pressure. As the air inlet pressure increases, the crushing position moves forward. When the air inlet pressure is $0.5 \mathrm{MPa}$, the crushing occurs first. Under the condition of constant water injection pressure, it can be seen from Fig. 6 that the injection depth decreases gradually with increasing air inlet pressure. When the air inlet pressure is $0.5 \mathrm{MPa}$, the injection depth is the minimum. Under the influence of high-pressure airflow, the liquid jet rapidly decomposes into small droplets of different sizes, which are mainly concentrated in the front area of the jet.
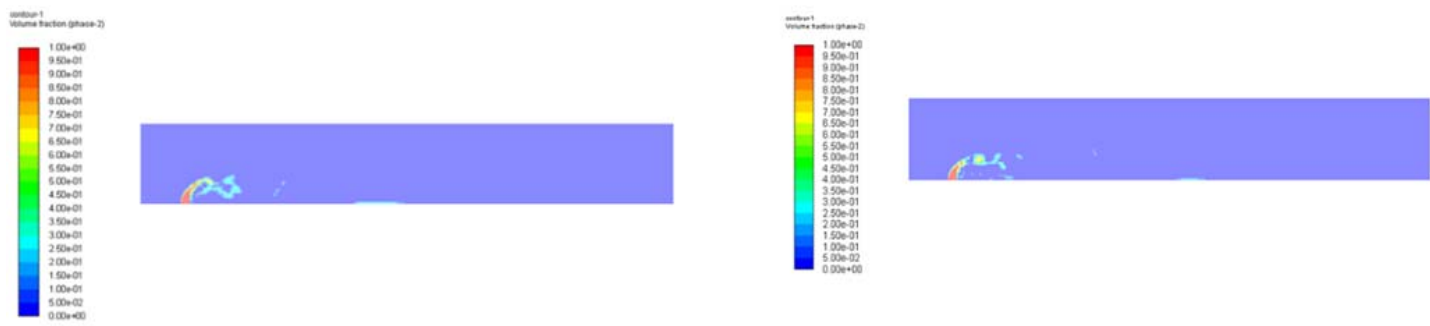

(a)Air inlet pressure: $0.2 \mathrm{MPa}$

(b) Air inlet pressure: $0.3 \mathrm{MPa}$
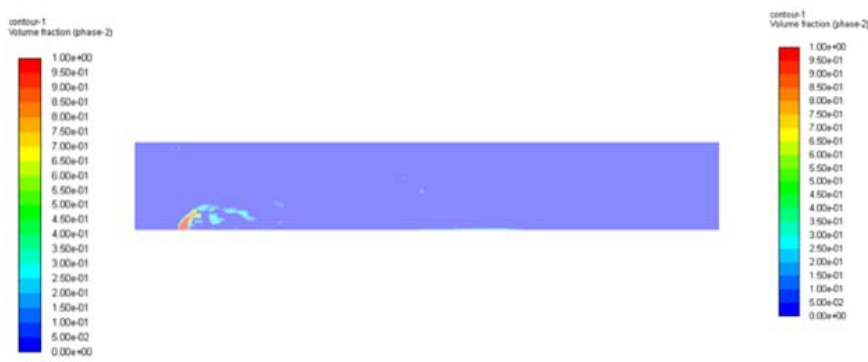

(c) Air inlet pressure: $0.4 \mathrm{MPa}$

(d) Air inlet pressure: $0.5 \mathrm{MPa}$

Fig.5. Injection depth distribution at different air inlet velocities.

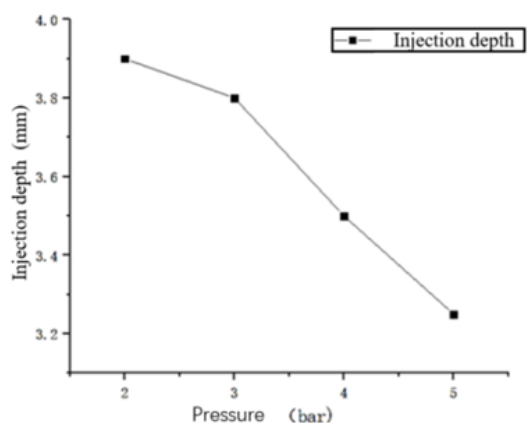

Fig.6. Influence of air inlet pressure on injection depth.

\section{Experimental verification}

\subsection{The experimental device}

The test device mainly includes a computer, mesh wire, charger, trigger, and high-speed 
camera. The test platform was built according to the test requirements. The AOS Technologies industrial camera was used for the high-speed camera. Under the condition of full resolution, the maximum shooting speed of this camera is more than 1000FPS. The image sensor type is CMOS, and it has a multi-head analysis system composed of two independent cameras to ensure the accuracy of the test.

\subsection{The nozzle set}

The corrosion inhibitor solution used in the injection-type jet device was mixed with abrasive and compressed air before the inlet end of the nozzle, the injection-type jet device was chosen to let the dust and abrasive wetted more fully and better effect of dust pollution control. The scavenger system and injection-type jet device is shown in Fig.7.
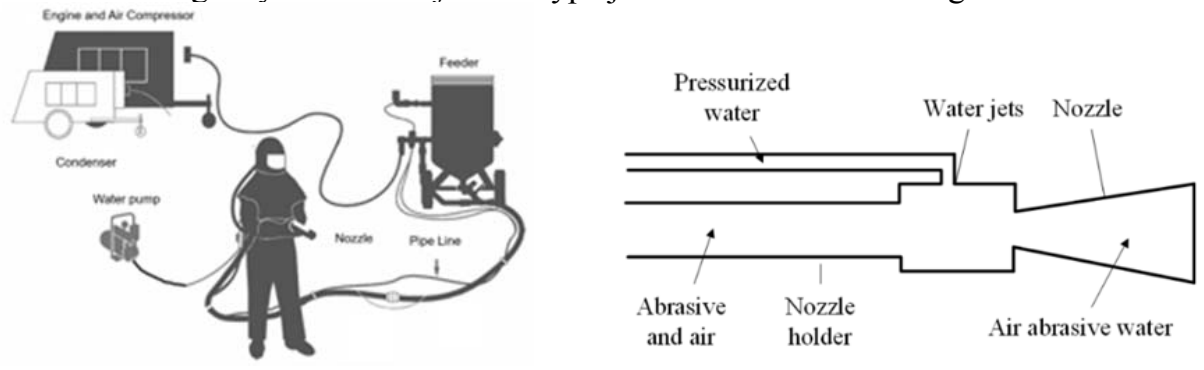

Fig.7. The scavenger system and injection-type jet device

\subsection{External atomization characteristics of the nozzle}

The test results of four groups, were similar, so only one of them was selected for comparison. As shown in Fig.8, with increasing the air inlet pressure, the atomization divergence area increases, and the atomized water droplets will become smaller. When the air inlet pressure is $0.2 \mathrm{MPa}$, the atomization distribution at both ends of the flat nozzle is not uniform, the spray inside is mainly composed of atomized water droplets, and the dust removal efficiency is poor. When the air inlet pressure is $0.3 \mathrm{MPa}$, the atomization state and dust removal effect are improved. With the constant increase of air inlet pressure, the external atomization state of the nozzle is good, and the dust removal effect is significantly improved. When the air pressure is $0.5 \mathrm{MPa}$, the dust removal effect is the best, which is the parameter with higher cleaning efficiency. 


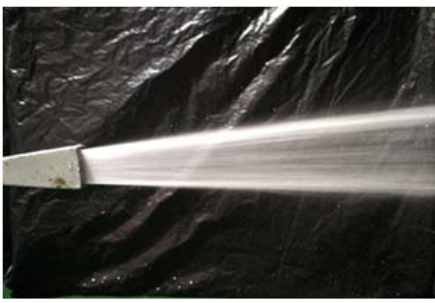

(a) Air inlet pressure: $0.2 \mathrm{MPa}$

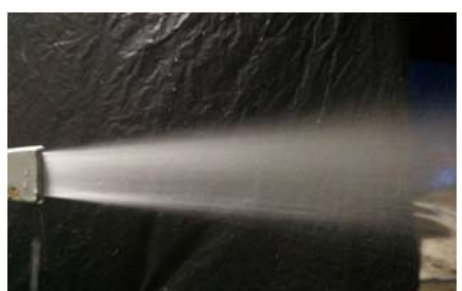

(c) Air inlet pressure: $0.4 \mathrm{MPa}$

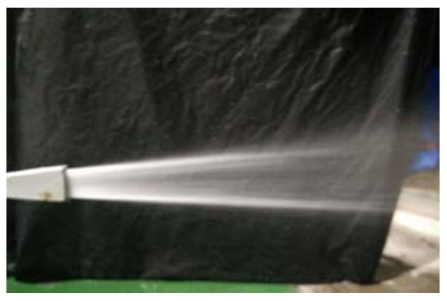

(b) Air inlet pressure: $0.3 \mathrm{MPa}$

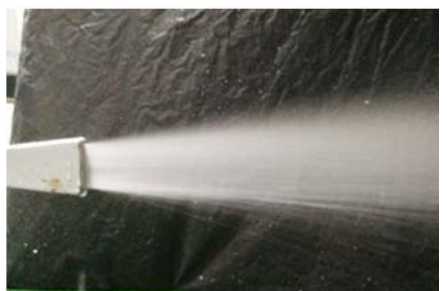

(d) Air inlet pressure: $0.5 \mathrm{MPa}$

Fig.8. Front atomization of the nozzle.

In the process of dry powder cleaning, when the particles impact on the glass surface, the particles will then be broken, rebound, and broken particles are the main cause of dust in the process of cleaning. Water diversion atomization suppress the broken particles, leading to achieve the purpose of dust removal.

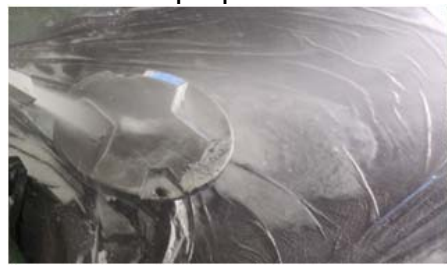

(a) $0.1 \mathrm{~s}$

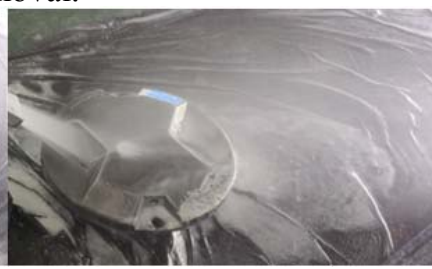

(b) $0.2 \mathrm{~s}$

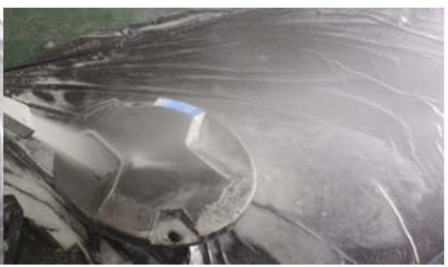

(c) $0.3 \mathrm{~s}$

Fig.9. The effect of no water in the cleaning process.

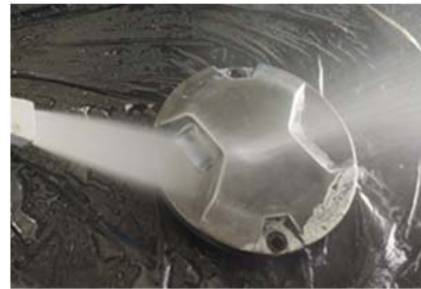

(a) $0.1 \mathrm{~s}$

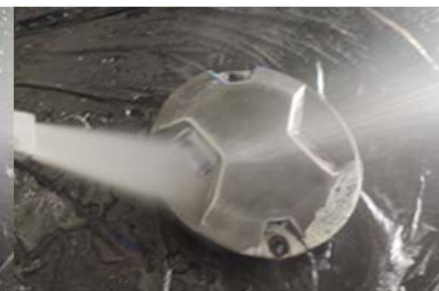

(b) $0.2 \mathrm{~s}$

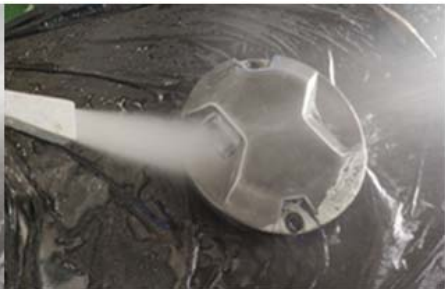

(c) $0.3 \mathrm{~s}$

Fig.10. Dust removal effect in the cleaning process.

The optimized parameters for experimental observation were used to compare with the test results as shown in Fig.9. In Fig.9, when water was not added for cleaning, particles were irregularly bounced around the cleaning environment. In Fig.10, when water was added for cleaning, the bounced broken particles combined with the surrounding water mist, and the particles were wetted, thus reducing the spatter ability and removing about $90 \%$ dust. For the unbroken particles, because the particle volume was large and thus difficult to be wet by water mist, the particles splashed around the cleaning environment. According to the comparative analysis of Fig.9 and Fig.10, in the process of dry powder cleaning, the external 
atomization of the nozzle can effectively suppress the broken particles. When the injection pressure is $0.5 \mathrm{MPa}$, the atomization dust removal effect is good. The unbroken particles will dissolve in water when washed in clean water.

\section{Conclusion}

1. According to the simulation analysis results of the CLSVOF method and the theory of liquid jet in transverse flow, it is concluded that the jet breaking position moves forward gradually with increasing the air inlet pressure.

2. The injection depth decreases with increasing the air inlet pressure.

3. For the external atomization state and dust removal effect of the nozzle, the experimental observation showed that when the air inlet pressure is $0.5 \mathrm{Mpa}$, the atomization state and dust removal effect of the nozzle are the best, effectively reducing the harm of dust to the human body.

\section{Acknowledgements}

This work was supported by the the Foundation of External Cooperation Project in Fujian Province, China (GrantNo.2020I0027); the Natural Science Foundation of Fujian Province, China(GrantNo.2020J01289); the open fund of Fujian Provincial Key Laboratory of Functional Materials and Applications (Xiamen University of Technology), grant number fma2020002; the Program for Innovative Research Team in Science and Technology in Fujian Province University (Xiamen University of Technology).

\section{References}

1. Hu Weiwei. Development of Dry Powder Cleaning System for Vehicle Airport Runway Guide Light [D]. Xiamen University of Technology,2017.

2. Wang Xing, GE Xiaohong, Yi Mingyang, Xu Yanhui. Journal of Xiamen University of Technology,2018,26(03):20-24.

3. Gao Qingji, Wang Wenzhou, Wang Qian, Zhu Wangzhen. Journal of civil aviation university of China,2019,37(02):49-52.

4. Cheng Binbin, Yu Zhijing. Mechanism of pollution rubber layer removal of embedded airport navigation aids[J].Science Technology and Engineering,2019,19(02):86-90.

5. A. Momber. Blast Cleaning Technology[M].Springer-Verlag Berlin Heidelberg, 2008.

6. Ohnesorge W. Formation of Drops by Nozzles and the Breakup of Liquid Jets [J].ZeitschriftfuerAngewandte Mathematic und Mechanik, 16:355-358,1936.

7. Reitz, R. D., Atomization and Other Breakup Regimes of a Liquid Jet[J].Ph.D. thesis, Princeton University, Princeton, NJ, 1978. 